\section{THE ROLE OF GRAIN BOUNDARY SLIDING ON CREEP DEFORMATION CHARACTERISTICS OF DISCONTINUOUS REINFORCED COMPOSITES}

\author{
S.B. Biner \\ Ames Laboratory \\ Iowa State University \\ Ames, IA 50011 U.S.A
}

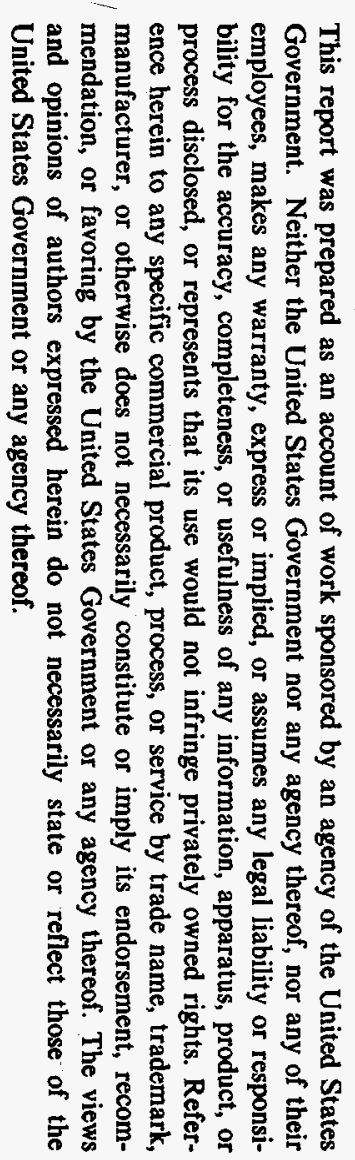

\section{ABSTRACT}

In this study, the grain boundary sliding behavior in discontinuous reinforced composites is investigated numerically. The results indicate that the stress enhancement factor for the composite is much larger than the one observed for the matrix material. In the composite, the increase in the strain rates as a result of grain boundary sliding occurs in a wider stress range in comparison to the matrix. It will be shown that the experimentally observed large scale triple point grain boundary cavitation in the composites could occur as a result of large grain rotations resulting from grain boundary sliding and evolution of triaxial stress state. Also, the observed larger creep exponent values or stress dependent creep exponent values for the composites may not be explained solely by the mechanism of grain boundary sliding.

\section{INTRODUCTION}

Several effects of grain boundary sliding on the deformation behavior of polycrystalline solids at creep regime have been widely recognized. First, fundamental considerations demand that the stress-strain rate relation plotted on $\log$-log coordinates should show an Sshaped curve as schematically shown in Fig. 1. The creep strain rate becomes slightly higher in the low stress, low strain rate regime as a result of grain boundary sliding (1). If the creep constitutive equation for non sliding homogenous polycrystalline solids can be characterized by the power law equation

$$
\dot{\varepsilon}_{c}=\dot{\varepsilon}_{o}\left(\frac{\sigma_{e}}{\sigma_{o}}\right)^{n}
$$

where $\sigma_{o}$ is a reference stress, $\dot{\varepsilon}_{o}$ is a temperature dependent reference strain rate, $n$ is the creep exponent and $\sigma_{e}$ is the equivalent stress. For sliding grain boundaries the corresponding relation is 


\section{DISCLAIMER}

Portions of this document may be illegible in electronic image products. Images are produced from the best available original document. 


$$
\dot{\varepsilon}_{c}=\dot{\varepsilon}_{o}\left(f \frac{\sigma_{e}}{\sigma_{o}}\right)^{n}
$$

where $f(f>1)$ is usually called the stress enhancement factor. The stress enhancement factors for polycrystalline solids have been determined by several investigators (2-5). From these analyses, it appears that $f$ does not depend on the grain size, but may depend on the geometrical parameters related to grain shape. Although in an earlier analysis (2) $f$ values were found to be independent of the creep exponent $n$ (having values of $1.1 \mp 0.01$ for $1 \leq n$ $\leq 8.8$ ), more recent studies $(3,5)$ have shown a larger value for $f$ and a slight increase in $f$ with increasing creep exponent ( 1.1 to 1.26 for $1 \leq n \leq 8)$.

It has been conclusively shown that for cavity nucleation at the grain boundaries as a result of development of high interface stresses, grain boundary sliding is essential $(6,7)$. Because the formation of facet cracks is associated with the growth and coalescence of nucleated grain boundary cavities, the growth mechanism of nucleated cavities has been studied extensively (8-11); and it has been shown that the cavity growth rate is substantially higher in the presence of grain boundary sliding (12-14). Finally, a crack in a homogeneous body under a compressive stress parallel to the crack plane will produce no perturbation in the absence of grain boundary sliding. However, in the presence of grain boundary sliding the same crack will be wedged open and stress-strain fields will be disturbed by the crack. Therefore, the intergranular creep fracture with grain boundary sliding will be more sensitive to the stress triaxiallity (5), and the tertiary creep range will also be strongly influenced by the presence of grain boundary sliding (13). The creep deformation behavior of composites significantly differs from that of polycrystalline matrix material. Although the rupture times are much longer in the composites, they usually exhibit larger creep exponent or stress dependent creep exponent values (i.e., $n$ value changes above a certain stress level) (15-18). In composites a much larger scale creep cavitation at the triple grain boundaries has also been reported (16-18).

In this study, in order to elucidate the grain boundary sliding behavior in discontinuously reinforced composites, series of numerical analyses were carried out. The results obtained were compared to grain boundary sliding behavior in a polycrystalline matrix having identical grain morphology.

\section{THE DETAILS OF THE NUMERICAL ANALYSIS}

In the present analyses the matrix and the composite behavior are investigated separately. The matrix was modelled as a unit-cell of an array of two dimensional grains as shown in Fig. 2a. On the other hand, for the composite with a $20 \%$ volume fraction reinforcement, a unit-cell around the periodic array of aligned discontinuous fibers within the two dimensional grains as shown in Fig. $2 b$ was employed. The deformation of the unit-cell must be constrained to maintain the compatibility and equilibrium with the adjacent material. This constraint requires that the cell boundaries remain straight and orthogonal and free of shear traction. Several methods for imposing these requirements on the FEM models have been suggested. The procedure outlined in (19) was utilized in this study.

In both cases the grain sizes and the grain shapes were identical. In order to clearly reveal the grain structure, the four triangular linear displacement elements which build up the quadrilateral elements have been omitted from Fig. 2. The grain boundaries were considered to be free to slide. However, in the analyses of the composite behavior only the grains next to the reinforcement were assumed to be rigidly connected to the reinforcement. To simulate the free boundary sliding, the corresponding nodes on both sides of a grain boundary were 
constrained to have the same displacements in the direction normal to the boundary but were otherwise free to move in the local tangential direction. The nodes of three adjacent grains at triple points were pinned together to meet the compatibility conditions.

An incremental large displacement FEM program has been developed which follows the formulation of Kanchi et al. (20). In the usual manner for problems involving non-linear continuum solids, it is assumed that the total strain $\varepsilon$ can be separated into an elastic component $\varepsilon_{e}$ and a creep component $\varepsilon_{c}$, i.e.,

$$
\varepsilon=\varepsilon_{e}+\varepsilon_{c}
$$

The elastic strain increments in any time interval are obtained by subtracting the creep strains from the total strain increments. These are multiplied by the elasticity matrix $D$ to give the stress increments:

$$
\Delta \sigma_{n}=D \Delta \varepsilon^{n}=D\left(\Delta \varepsilon-\Delta \varepsilon_{\varepsilon}^{n}\right)
$$

The grain interior deformations taken are to be described by classical $J_{2}$ flow theory. The strain increment $\Delta \varepsilon_{C}^{n}$ occurring in a time interval $\Delta \mathrm{t}=\mathrm{t}_{n+1} \mathrm{t}_{n}$ can be approximated by

$$
\Delta \varepsilon_{c}^{n}=\Delta t_{n}\left[(1-\theta) \varepsilon_{c}^{n}+\theta \varepsilon_{c}^{n+1}\right]
$$

For $\theta=0$ the algorithm is "fully explicit", while $\theta=1$ gives a "fully implicit" algorithm. During any time increment the equilibrium equations which must be satisfied are (in incremental form)

$$
\int_{V}[\mathrm{~B}]^{T} \Delta \sigma_{n} \mathrm{dV}+K_{T}^{n} \Delta U_{n}-\Delta R_{n}=0
$$

Here $K_{T}^{n}$ is the tangential stiffness matrix, $[B]^{T}$ is the strain displacement matrix, and $\Delta R_{n}$ is the external load increment during the step (here $\Delta R_{n}=0$ for all time steps other than the first increment). Since the stress increment calculation is based on the linearized form of the incremental equilibrium equation, it does not always satisfy the equation of equilibrium exactly. Thus, when the stresses $\sigma_{n+1}=\sigma_{n}+\Delta \sigma_{n}$ are substituted back into equation of equilibrium, residual forces remain. These are then added to the applied force increment at the next iteration solution. For this study, an implicit time stepping procedure with $\theta=1$ was used with variable time increments. The time steps were chosen according to the stability criterion proposed by Cormeau (21) in order to avoid numerical instabilities.

During the analyses the creep behavior of the interior of the grains was assurned to obey eq. 1. The value of $n$ was taken as $n=2$ and $1 / \varepsilon_{o}$ provides a natural time scale during the solution. Furthermore, Young's modulus relating the elastic strain increments in the matrix was taken as $E_{m}=10^{3} \sigma_{0}$. In the case of the composites the Young's modulus of the reinforcement was assumed to be $E_{f}=5 E_{m}$ and remained elastic during the course of creep deformation. For both the matrix and the reinforcement, Poisson's ratio was chosen as $v=0.3$.

To simulate the constant-load creep test, normal traction was applied at the beginning of the solution to the end of the unit-cells such that the area integral of the traction was equal to the applied load: 


$$
\int_{\mathrm{A}} T_{i} \mathrm{dA}=\sigma \mathrm{A}
$$

\section{RESULTS AND DISCUSSION}

The results for the evolution of the stress fields during the initial loading (i.e., at time $t=0$ ) in the composite and inside the sliding grains in a polycrystalline matrix material will be given first. The distribution of the stress components and resulting hydrostatic stress in the composite at the continuum scale are shown in Fig. 3. The axial stress carried by the reinforcement is much larger than that carried by the matrix; on the other hand, near the reinforcement ends within the matrix, the development of the large axial stress component caused by the stress concentration effect of the sharp corners of the reinforcement can be seen in Fig. 3a. Although the applied stress was uniaxial tension, transverse stress develops in the composite and its distribution differs both quantitatively and qualitatively from that of the axial stress component. As can be seen from Fig. $3 \mathrm{~b}$, this stress component takes on a negative value between the reinforcements, while the regions at the tip of the reinforcements exhibit a positive value. The resulting nonuniform distribution of hydrostatic stress is given in Fig. 3c. As discussed in an earlier study (22), the creep hardening in discontinuous reinforced composites deforming with a power-law is associated with this buildup in the hydrostatic stress which lowers the equivalent stress level. Of course these stress distributions are strong functions of the parameters of the unit-cell and aspect ratio of the reinforcement and vary considerably with the variations in these parameters (22).

The distribution of different stress components within the grains of a polycrystalline material (Fig. 2a) is given in Fig. 4. Due to grain boundary sliding, the deformation is no longer homogeneous throughout the grains even though the applied stress is uniaxial tension. As can be seen from Fig. 4 for all stress components, the distribution is periodic with the extreme values occuring at the triple points. The contour values clearly show the positive and negative stress concentrations at the 60 and 120 degree sectors of the grain boundary. Although it is not resolved in Fig. 4, all the stress components are singular at the triple points as determined by asymptotic analysis (23). This distribution of the stress components will of course be different for virtually each grain depending on its location in the unit-cell of the composite due to the variation of stresses at the continuum scale (Fig. 3).

The variations of the steady state creep rates for the polycrystalline matrix are given in Fig. 5. As can be seen, for low stress level and low strain rate regime the creep strain rate is higher and the observed value agrees well with the estimated value by using stress enhancement factor, $f$, of 1.14 for $n=2(3,5)$ as indicated in the figure. With increasing stress, the contribution of grain boundary sliding to the creep deformation rate becomes smaller, and the observed steady state creep rates agree with the values predicted from the creep constitutive equation (eq. 1). The stress range in which this transition occurs can also be seen from the figure.

For the case of the composite, the initial analyses were carried out at the continuum scale in order to elucidate the creep behavior in the absence of grain boundary sliding. These results are compared with the behavior of the matrix material in Fig. 6. The observed creep rates in the absence of grain boundary sliding are almost an order of magnitude smaller for the composite than the creep rates observed for the matrix material. This behavior is associated with the development of triaxial stress state as discussed earlier (Fig. 3). In the following simulations, the observed steady state creep rates for the composite with sliding grain boundaries are summarized in Fig. 6. It is apparent that the contribution of the grain boundary sliding to creep deformation rate is much larger in the composite in comparison to the matrix material. The stress enhancement factor for the composite was found to be 1.86 in comparison to a value of 1.14 for the matrix material. In contrast to polycrystalline matrix 
material, in the composite this contribution occurred in all stress ranges investigated, and absence of a transition region can also be seen in Fig. 6 . Furthermore, the grain boundary sliding did not significantly change the creep exponent of the composite. This result indicates that the experimentally observed larger creep exponent values or stress dependent creep exponent values for the composites cannot be explained solely with the grain boundary sliding mechanism.

The typical creep displacement fields under a far field uniform tensile stress for a polycrystalline matrix material (Fig. 2a) are shown in Fig. 7. The grain boundary sliding on the vertical grain boundaries with broken horizontal lines can be easily identified. In contrast, absence of grain boundary sliding on the horizontal grain boundaries can be seen from the continuity of the vertical lines. The overall creep displacement field in the composite at identical applied tensile stress level is shown in Fig. 8, and an enlarged section around the reinforcement is given in Fig. 9. The resulting nonuniform displacement field in the composite can be clearly seen in Fig. 8 . While the grains around the reinforcement undergo large rotations as a result of vertical and horizontal grain boundary sliding, at regions away from the reinforcement the sliding behavior was more uniform and similar to that shown in Fig. 7 for the matrix material. The resulting cavitation at the triple points as a result of this unaccommodated grain rotation for sliding grain boundaries can be seen in Fig. 9. This result clearly indicates one of the possible reasons for the experimentally observed high degree of grain boundary cavitation in the composites (16-18).

The present study of grain boundary sliding effects employing two dimensional planestrain hexagonal grains is clearly only an approximation of real polycrystalline material. Also, in the unit-cell analysis of the composite behavior the assumption of fully aligned reinforcement and completely periodic pattern of distribution are an idealization. The thermal stresses due to the differences in thermal expansion coefficients of the phases were also neglected during the analysis. Rodin and Dib (4) have performed FEM analysis for determination of stress enhancement factor using three dimensional Wigner-Seitz unit-cells for a polycrystalline material. Their analyses have shown larger values of stress enhancement factor but decreasing behavior with increasing creep exponent (1.31 to 1.23 for $1 \leq n \leq 7$ ). Although the stress enhancement factor has been evaluated by several authors (1-5) for different grain geometries, the characterization of it by a proper parameter has not been very successful $(4,5)$. In spite of the above mentioned simplifications, present results clearly show the differences in the grain boundary sliding behavior between a polycrystalline matrix material and a discontinuous reinforced composite both having identical grain morphology. Although stress enhancement factor is independent of the grain size for a polycrystalline material, it is expected that the observed differences will be influenced by the grain size in the composite because of the degree of grain rotation and also by the geometric parameters of the reinforcement which alter the triaxial stress state (22).

\section{CONCLUSIONS}

In this study grain boundary sliding behavior in discontinuously reinforced composite and a polycrystalline matrix having identical grain morphologies was investigated. The results indicate that:

1. The stress enhancement factor for the composite is much larger than the one observed for the matrix material.

2. In the composite, the increase in the strain rates as a result of grain boundary sliding occurs in a wider stress range in comparison to the matrix.

3. The experimentally observed large scale triple point grain boundary cavitation in the composites could occur as a result of large grain rotations resulting from grain boundary sliding and evolution of triaxial stress state. 
4. Experimentaly observed larger creep exponent values or stress dependent creep exponent values for the composites may not be explained solely by the mechanism of grain boundary sliding.

\section{ACKNOWLEDGEMENTS}

This work was performed for the United States Department of Energy by Iowa State University under contract W-7405-Eng-82. This research was supported by the Director of Energy Research, Office of Basic Energy Sciences.

\section{REFERENCES}

1. I. W. Chen and A. S. Argon, Grain boundary sliding and interphase boundary sliding in power law creep, Acta Metall. 27:749 (1979).

2. F. W. Crossman and M. F. Ashby, The non-uniform flow of polycrystals by grain boundary sliding accommodated by power law creep, Acta Metall. 23:425 (1975).

3. F. Ghahremani, Effect of grain boundary sliding on steady state creep of polycrystals, Int. J. Solids Struct. 16:847 (1980).

4. G. J. Rodin and M. W. Dibb, Effective properties of creeping solids undergoing grain boundary sliding, in : "Advances in Fracture Research," K. Salame et. al., eds., Pergoman Press, Oxford (1989).

5. K. J. Hsia, D. M. Parks and A. S. Argon, Effects of grain boundary sliding on creep constrained boundary cavitation and creep deformation, Mech. of Materials 11:43 (1991).

6. A. S. Argon, Mechanics and mechanism of fracture in creeping alloys, in: "Recent advances in creep and fracture of engineering materials and structures," B. Wilshire et. al., eds., Pineridge Press, Swansea (1982).

7. H. Riedel, Cavity nucleation at particles on sliding grain boundaries. A shear crack model for grain boundary sliding in creeping polycrystals, Acta Metall. 32:313 (1984).

8. B. F. Dyson and M. F. Ashby, Creep damage mechanics and micromechanics, in: "Ancvances in Fracture Research 84," S. R. Valluri et.al., eds., Pergamon Press, Oxford (1984).

9. I. W. Chen and A. S. Argon, Diffusive growth of grain boundary cavities, Acta Metall. 29:1759 (1981).

10. V. Tvergaard, Constitutive relations for creep in polycrystals with grain boundary cavitation, Acta Metall. 32:1977 (1984).

11. J. R. Rice, Constraints on diffuse cavitation of isolated grain boundary facets in creeping polycrystal, Acta Metall. 29:675 (1981).

12. K. J. Hsia, D. M. Parks and A. S. Argon, Modelling creep damage evolution around blunt notches and sharp cracks, Mech. of Materials 11:19 (1991).

13. V. Tvergaard, Influence of grain boundary sliding on material failure in the tertiary creep range, int. $J$. Solids Struct. 21:279 (1985).

14. E. van der Giessen and V. Tvergaard, A creep rupture model accounting for cavitation at sliding grain boundaries, Int. J. Fractiure 48:153 (1991).

15. S. B. Biner and W. A. Spitzig, Creep characteristics of wire-drawn $\mathrm{Cu}-20 \% \mathrm{Nb}$, Mat. Sci. Eng. A150:213 (1992).

16. H. T. Lin and P. F. Becker, High temperature creep deformation of alumina SiC whisker composite, $J$. Am. Ceram. Soc. 74:1886 (1991).

17. T. G. Nieh, Creep rupture of a silicon carbide reinforced aluminum composite, Metall. Trans. 15A:139 (1984).

18. A. H. Chokshi and J. R. Porter, Creep deformation of an alumina matrix composite reinforced with silicon carbide whiskers, J. Am. Ceram. Soc. 68:C-144 (1985).

19. R. E. Smelser and R. Becker, Abaqus user subroutine for material modelling, in: "Abaqus user conference proceedings," (1990).

20. M. B. Kanchi, O. C. Zienkiewicz and D. R. J. Owen, The visco-plastic approach to problems of elasticity and creep involving geometric nonlinear effects, Int. J. Num. Meth. Eng. 9:109 (1975)

21. I. Cormeau, Numerical stability in quasistatic elasto-visco-plasticity, Int. J. Num. Meth. Eng. 9:109 (1975).

22. S. B. Biner, The role of fiber morphology on the creep behavior of discontinous fiber reinforced composites, Mat. Sci and Eng. A156:21 (1992).

23. C. W. Lau, A. S. Argon and F. A. McClintock, Stress concentrations due to sliding grain boundaries in creeping alloys, in: "Elastic-Plastic Fracture Mechanics," Am. Soc. Testing and Materials (1983). 


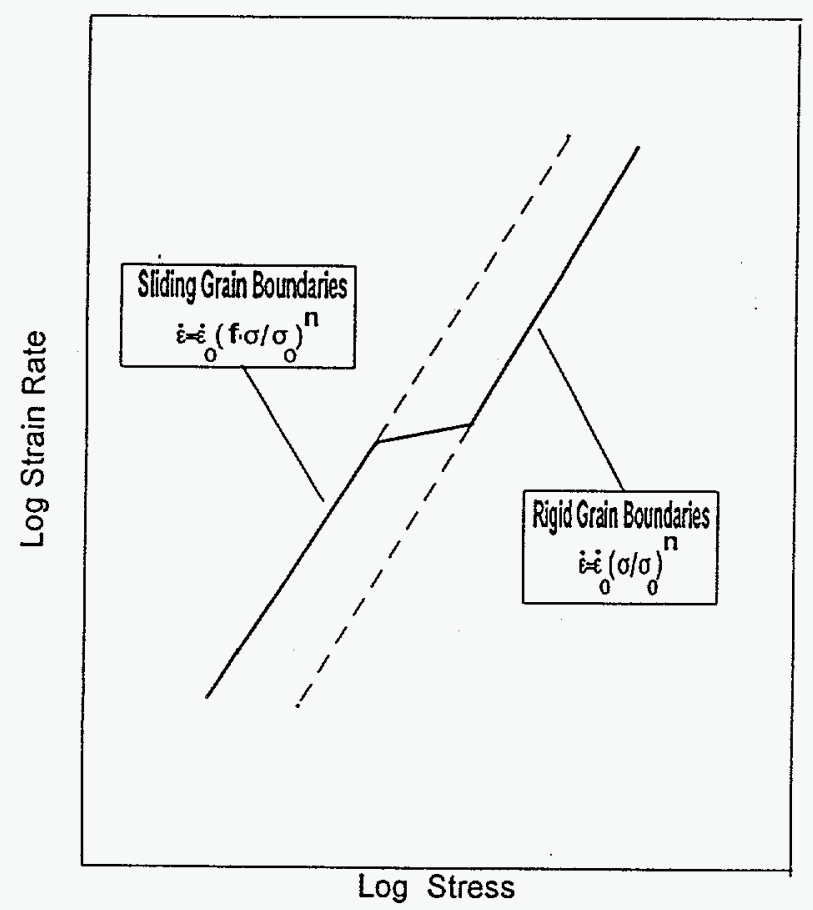

Fig. 1. Schematic representation of the role of grain boundary sliding on creep strain rates. 

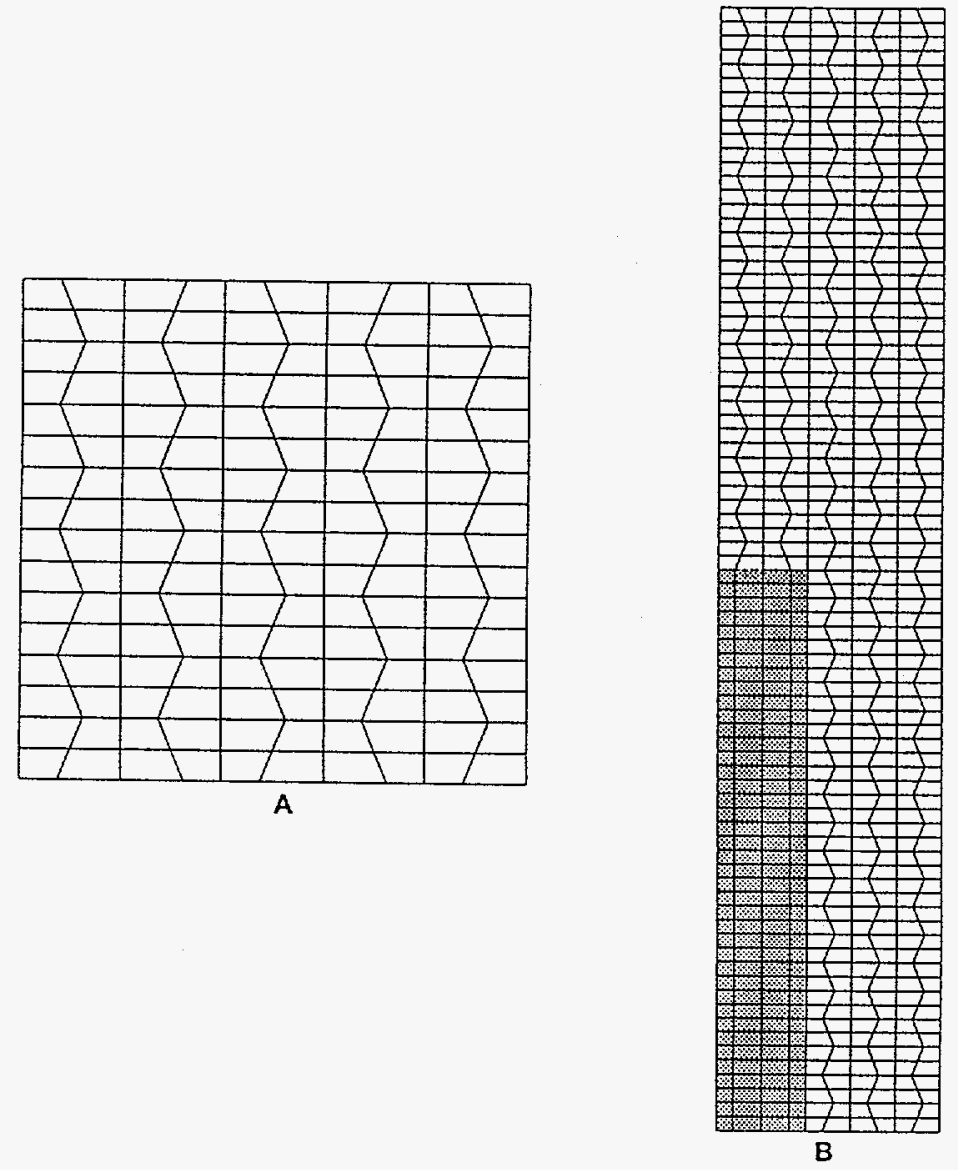

Fig. 2. The details of the grain structure used in the FEM analyses. a)- FEM model for polycrystalline matrix b)-FEM model for composite behavior, the dark region is the reinforcement. The four triangular linear elements that build up the quadrilateral elements were omitted in order to clearly reveal the grain morphology. 

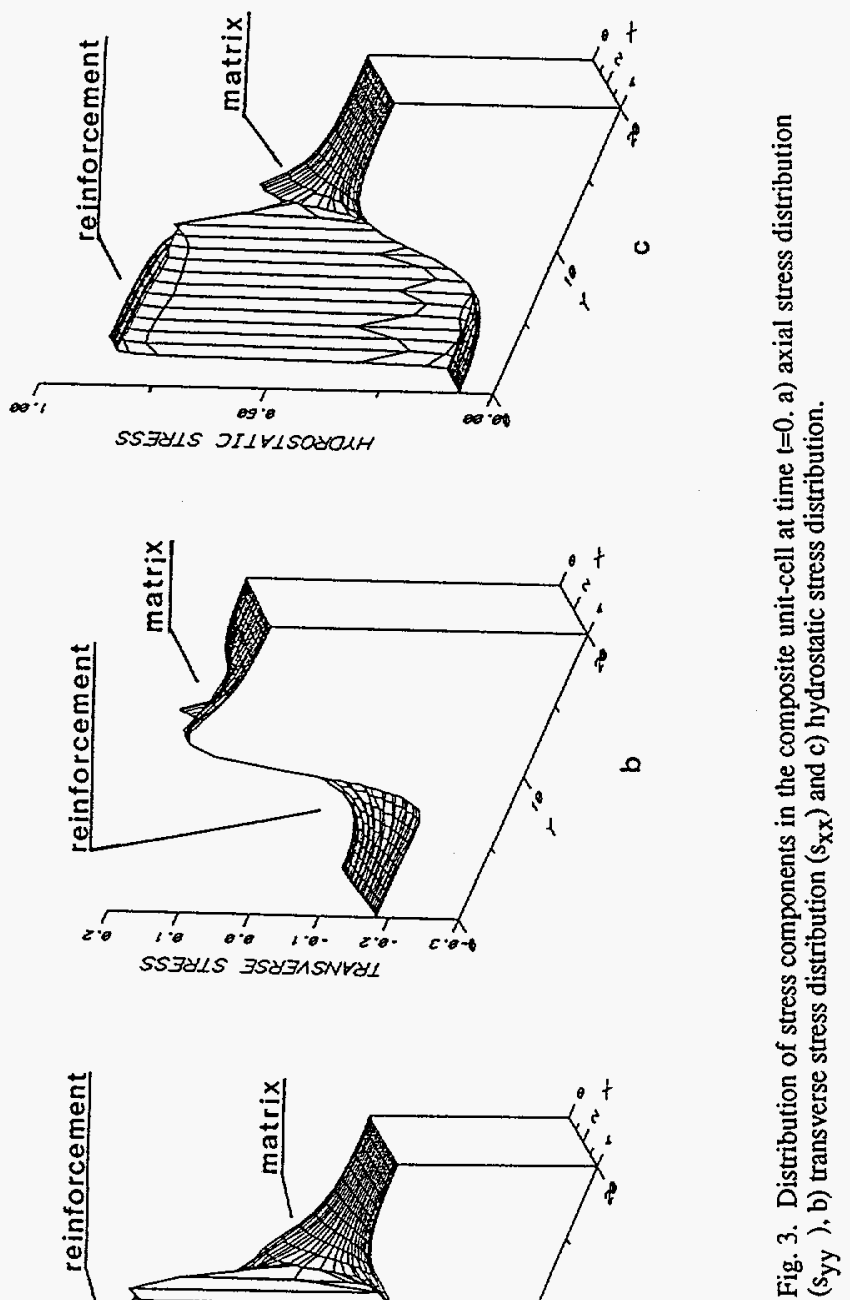


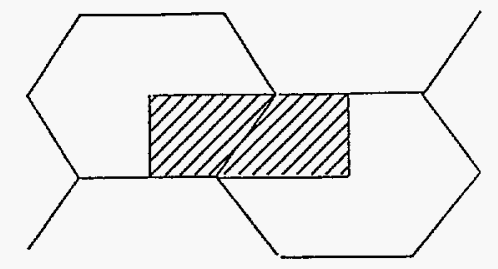

A

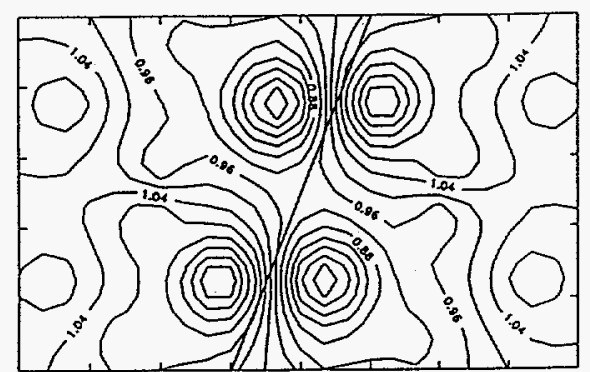

B

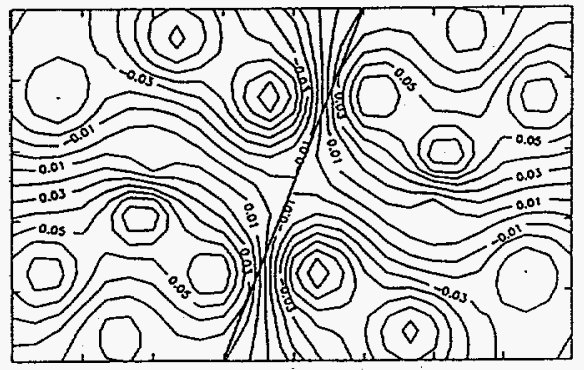

C

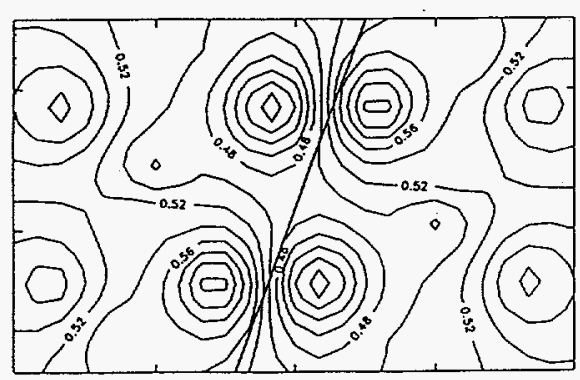

Fig. 4. Distribution of stress components in the grains of polycrystalline matrix with sliding grain boundaries a) axial stress distribution $\left.\left(s_{\mathrm{yy}}\right), \mathrm{b}\right)$ transverse stress distribution $\left(\mathrm{s}_{\mathrm{xx}}\right)$ and $\mathrm{c}$ ) equivalent stress distribution. 


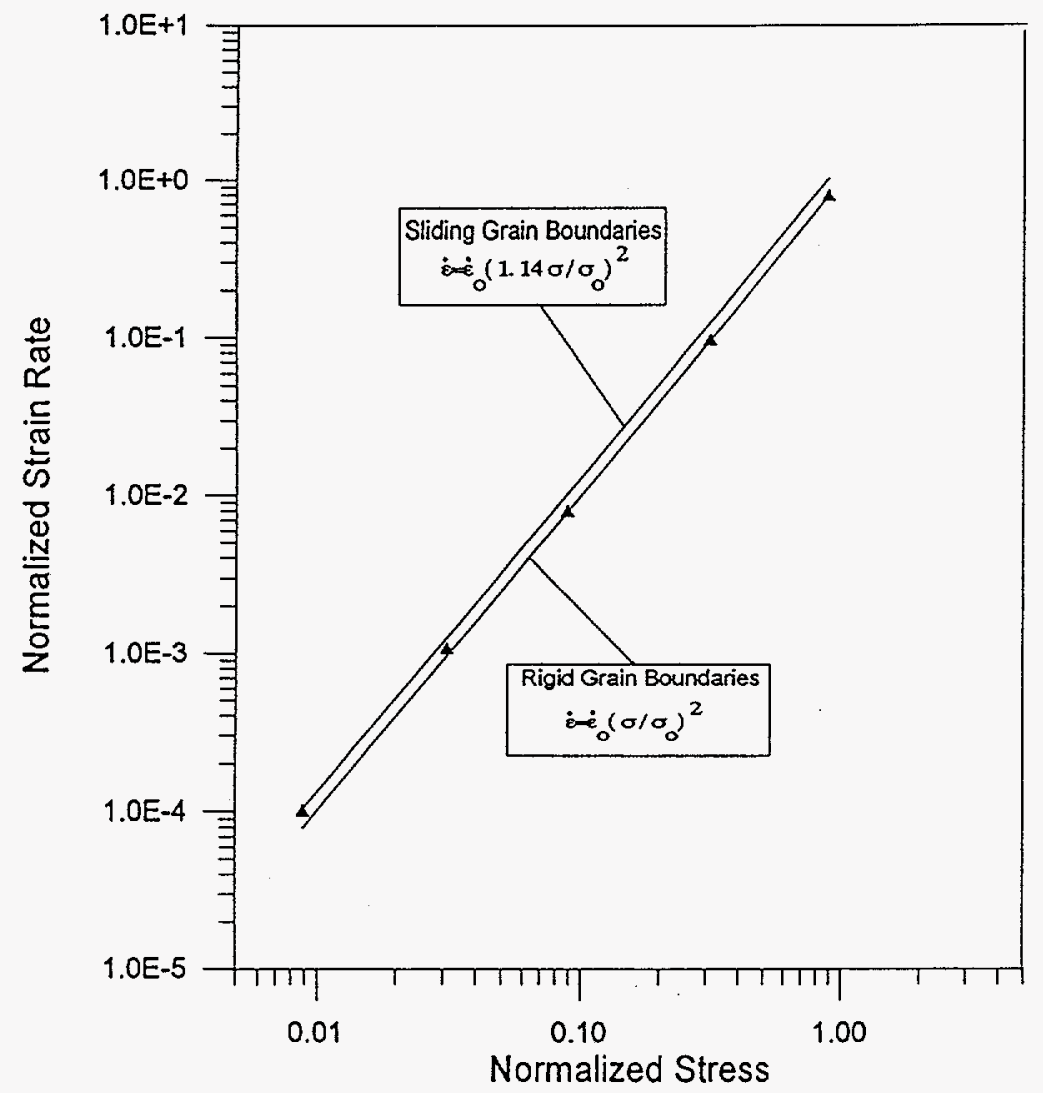

Fig. 5. Variation of steady state creep strain rate with stress for polycrystalline matrix with sliding grain boundaries. 


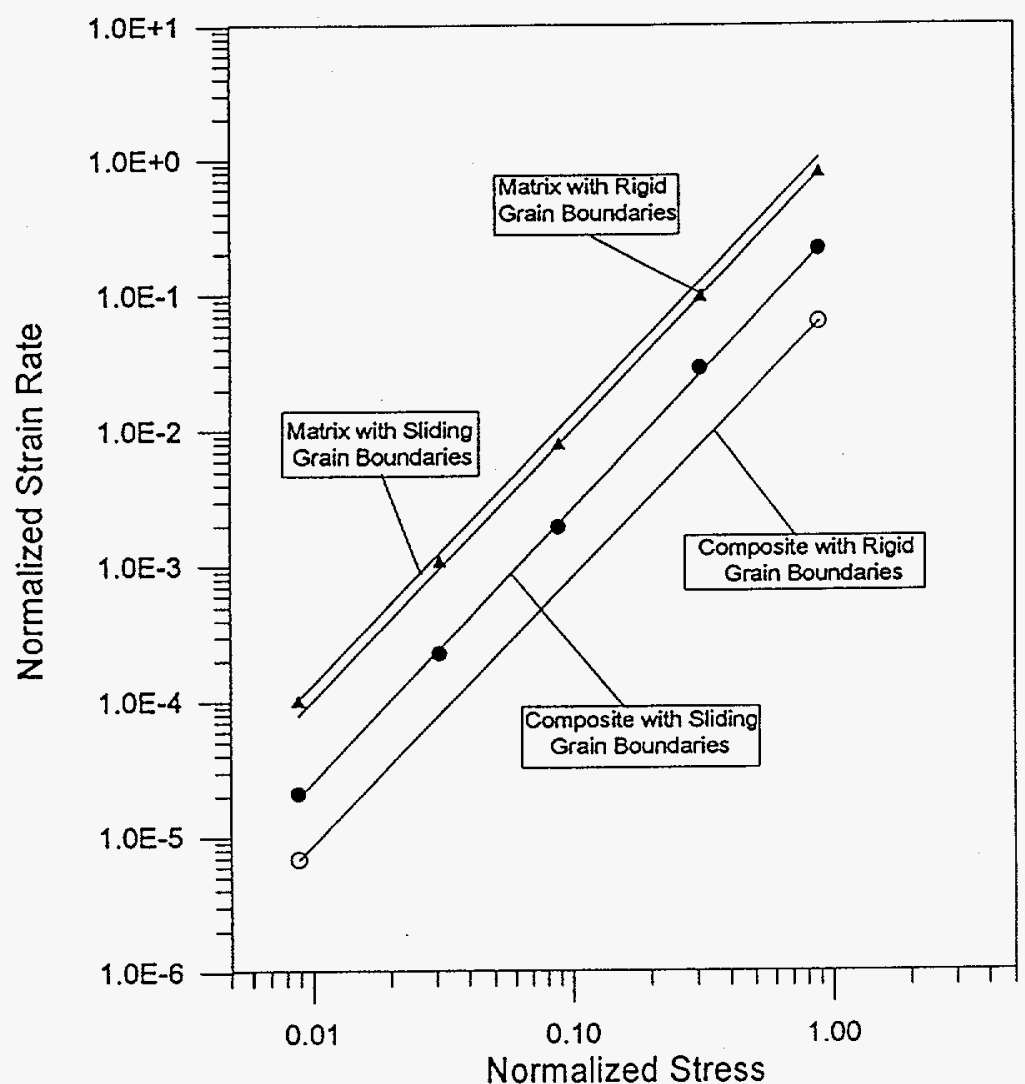

Fig. 6. Variation of steady state creep strain rate with stress for composites with sliding and non-sliding grain boundaries. 


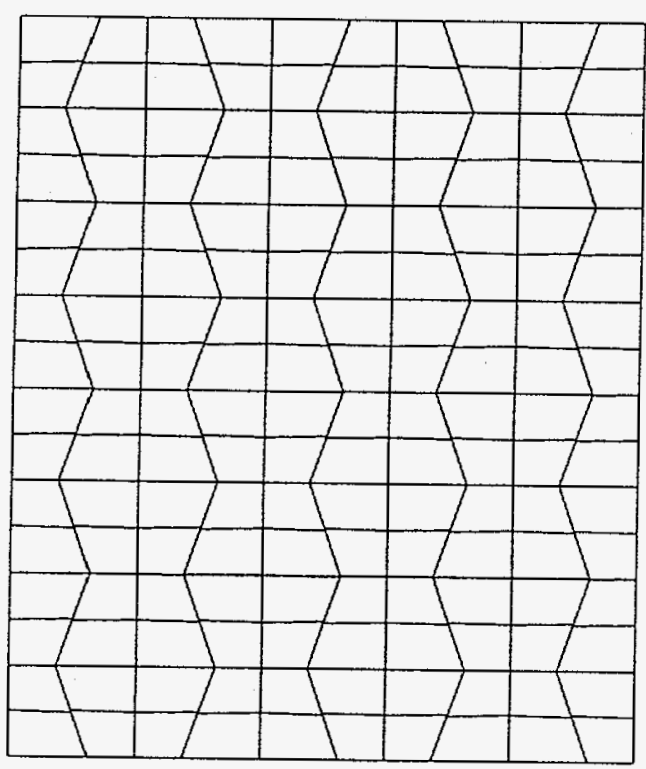

Fig. 7. Creep displacement field under a far field uniform tensile stress for a polycrystalline matrix. 


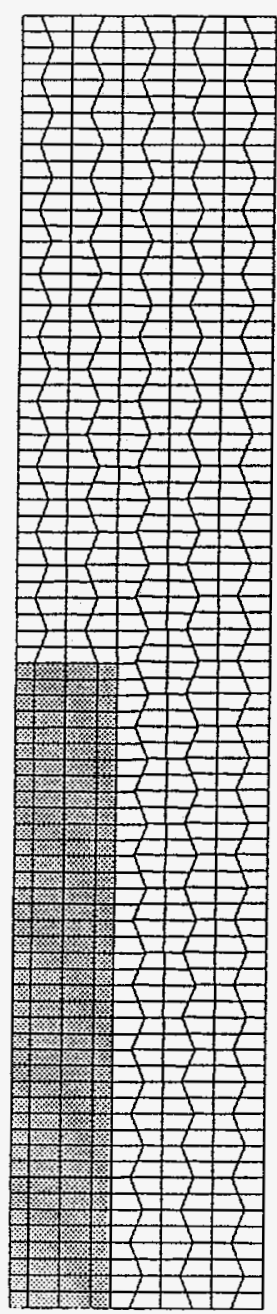

Fig. 8. Creep displacement field under a far field uniform tensile stress for composite. 


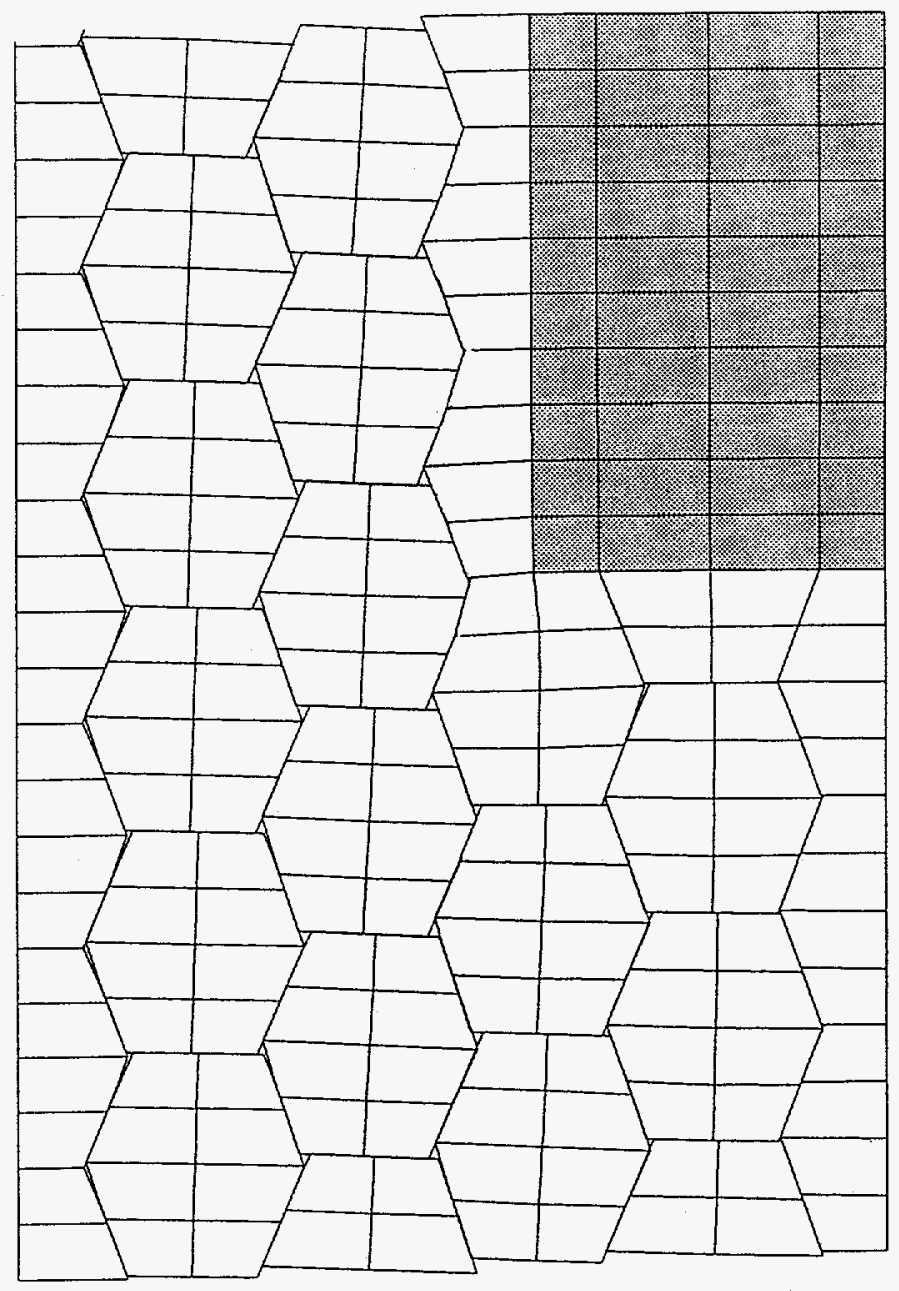

